\title{
Intrasaccadic perception triggers pupillary constriction
}

Sebastiaan Mathôt, Jean-Baptiste Melmi, Eric Castet

It is commonly believed that vision is impaired during saccadic eye movements. However, here we report that some visual stimuli are clearly visible during saccades, and trigger a constriction of the eye's pupil. Participants viewed sinusoid gratings that changed polarity 150 times per second (every $6.67 \mathrm{~ms}$ ). At this rate of flicker, the gratings were perceived as homogeneous surfaces while participants fixated. However, the flickering gratings contained ambiguous motion: rightward and leftward motion for vertical gratings; upward and downward motion for horizontal gratings. When participants made a saccade perpendicular to the gratings' orientation (e.g., a leftward saccade for a vertical grating), the eye's peak velocity matched the gratings' motion. As a result, the retinal image was approximately stable for a brief moment during the saccade, and this gave rise to an intrasaccadic percept: A normally invisible stimulus became visible when eye velocity was maximal. Our results confirm and extend previous studies by demonstrating intrasaccadic perception using a reflexive measure (pupillometry) that does not rely on subjective report. Our results further show that intrasaccadic perception affects all stages of visual processing, from the pupillary response to visual awareness. 
2 Title: Intrasaccadic Perception Triggers Pupillary Constriction

3 Authors: Sebastiaan Mathôt, Jean-Baptiste Melmi, and Eric Castet

4 Affiliation: Aix-Marseille University, CNRS, UMR 7290, Marseille, France

5 Manuscript ID: $4616(\mathrm{R} 1)$

6 This is a text-only manuscript. Figures have been uploaded separately and appear at the end

7 of the manuscript. Because the reviewing PDF is auto-generated, the line numbers as they appear

8 in the cover letter may not exactly correspond to the line numbers in the reviewing PDF. 
Abstract

10 It is commonly believed that vision is impaired during saccadic eye movements. However,

11 here we report that some visual stimuli are clearly visible during saccades, and trigger a

12 constriction of the eye's pupil. Participants viewed sinusoid gratings that changed polarity 150

13 times per second (every $6.67 \mathrm{~ms}$ ). At this rate of flicker, the gratings were perceived as

14 homogeneous surfaces while participants fixated. However, the flickering gratings contained

15 ambiguous motion: rightward and leftward motion for vertical gratings; upward and downward

16 motion for horizontal gratings. When participants made a saccade perpendicular to the gratings'

17 orientation (e.g., a leftward saccade for a vertical grating), the eye's peak velocity matched the

18 gratings' motion. As a result, the retinal image was approximately stable for a brief moment

19 during the saccade, and this gave rise to an intrasaccadic percept: A normally invisible stimulus

20 became visible when eye velocity was maximal. Our results confirm and extend previous studies

21 by demonstrating intrasaccadic perception using a reflexive measure (pupillometry) that does not

22 rely on subjective report. Our results further show that intrasaccadic perception affects all stages

23 of visual processing, from the pupillary response to visual awareness.

24 Keywords: pupillometry, eye movements, saccadic suppression, intrasaccadic perception 
When you look at yourself in the mirror, it seems that you make no eye movements. And that

27 is odd, because everyone else does.

28 The inability to see your own saccadic eye movements is a simple yet powerful

29 demonstration of saccadic suppression: You generally do not perceive events that occur while

30 your eyes are moving, including (tautologically) your own saccades. Phrased differently, the

31 retinal-image motion that results from saccades is suppressed, at least in the sense that it is not

32 consciously perceived. Saccadic suppression is sometimes considered a mechanism that prevents

33 you from seeing the world move when your eyes move (e.g., Ross, Burr, \& Morrone, 1996).

34 However, this functional interpretation is controversial (e.g. Castet, 2010; O'Regan \& Noë,

35 2001), and here we use saccadic suppression as an umbrella term for various findings that show

36 that vision is impaired around the time of saccades.

37 Saccadic suppression affects most if not all aspects of visual processing. Psychophysical

38 studies have shown that visual sensitivity is reduced during a $\pm 100 \mathrm{~ms}$ window around saccades

39 (Diamond, Ross, \& Morrone, 2000; Volkmann, Riggs, White, \& Moore, 1978; Zuber \& Stark,

40 1966), especially for non-isoluminant stimuli with a low spatial frequency (Burr, Morrone, \&

41 Ross, 1994; Knöll, Binda, Morrone, \& Bremmer, 2011)+(; but see Braun, Schuetz, \&

42 Gegenfurtner, 2015). Neurophysiological studies have shown that stimuli presented during that

43 same window elicit reduced neural activity in many visual brain areas (Bremmer, Kubischik,

44 Hoffmann, \& Krekelberg, 2009; Thiele, Henning, Kubischik, \& Hoffmann, 2002). Saccadic

45 suppression even affects the pupillary light response: The pupillary constriction that is triggered

46 by a flash of light is reduced when the flash occurs around the time of a saccade (Lorber, Zuber,

47 \& Stark, 1965; Zuber, Stark, \& Lorber, 1966).

48 However, saccadic suppression is not complete; sometimes you can see while the eyes move, 
49 a phenomenon that we will refer to as intrasaccadic perception. Intrasaccadic perception can

50 even be very conspicuous, as was elegantly shown by Campbell and Wurtz (1978). In their

51 experiment, participants sat in the dark and made a saccade. Crucially, if the experimental room

52 was illuminated during the saccade, participants perceived a smeared-out image of the room -an

53 intrasaccadic percept. But this only happened when the illumination period fell entirely within the

54 saccade. If the room was also briefly illuminated before or after the saccade, smearing was no

55 longer perceived; instead, participants perceived a static image of the room. In other words,

56 intrasaccadic perception occurred only when there was no pre- or postsaccadic visual stimulation;

57 otherwise, the intrasaccadic percept was not consciously perceived, presumably because it was

58 masked by the pre- and postsaccadic percept (reviewed in Castet, 2010). Intrasaccadic perception

59 has also been demonstrated with stimuli that move too rapidly to be seen with static eyes (e.g., a

60 vertical grating that moves across a display with a speed of $400 \%$ s). Saccades can make such

61 stimuli visible by reducing retinal-image motion: When you make a saccade in the direction of

62 the stimulus' movement (i.e. perpendicular to the grating's orientation), the velocity of the

63 saccade briefly reduces, or even cancels, the retinal speed of the stimulus, which is consequently

64 perceived as a static or moving percept, depending on the stimulus and the eye movement's

65 velocity (Castet \& Masson, 2000; Deubel \& Elsner, 1986; García-Pérez \& Peli, 2001). Phrased

66 differently, a saccade can make a normally invisible stimulus visible by briefly reducing, or even

67 canceling, its retinal speed. This is a striking phenomenon that we will use in the present study as

68 well. (We also provide demonstration software, as described under Methods.)

69 To date, intrasaccadic perception has only been demonstrated through subjective report. In a

70 typical experiment, participants first make a saccade, and then indicate whether or not they

71 perceived something (a flash or a movement) during the saccade. Here we use pupillometry to

72 provide a direct demonstration of intrasaccadic perception that does not rely on subjective report.

73 To this end, we used a paradigm similar to that used by Castet and Masson (2000), and measured 
74 pupillary responses, which are increasingly recognized as a powerful tool for vision science (for

75 recent reviews, see Binda \& Murray, 2014; Mathôt \& Van der Stigchel, 2015). Under normal

76 conditions, centrally presented visual stimuli trigger a pupillary constriction, even when there is

77 no change in overall luminance (e.g., Gamlin, Zhang, Harlow, \& Barbur, 1998; Slooter \& van

78 Norren, 1980; Ukai, 1985). Therefore, we predicted that an intrassacadic stimulus, which in our

79 view is not fundamentally different from any other stimulus, would also trigger a pupillary

80 constriction (or rather a strengthening of the pupillary constriction that is generally observed after

81 a saccade; Mathôt, van der Linden, Grainger, \& Vitu, 2015; Zuber et al., 1966).

82 If we would find a pupillary constriction to intrasaccadic perception, this would be important

83 for two main reasons. Firstly, our study avoids expectation effects that were not addressed

84 previously: The knowledge that you may see something during a saccade may by itself increase

85 the saliency of the intrasaccadic percept, and, in the extreme case, even induce a 'placebo

86 percept'. Therefore, we tested only naïve observers, and we did not inform them beforehand that

87 they might perceive 'something odd' during a saccade. Secondly, a pupillary constriction to

88 intrasaccadic perception would show that intrasaccadic perception is similar to regular

89 perception, not only in eliciting a conscious percept, but also in triggering a reflexive pupillary

90 response.

Methods

\section{Materials and availability}

93 Participant data, the experimental script, and analysis scripts are available from

94 https://github.com/smathot/materials_for_P0018. On-line materials include easy-to-use

95 demonstration software that allows anyone with a sufficiently fast display $(>=150 \mathrm{~Hz})$ to

96 experience intrasaccadic perception. 
Participants

98 Ten naïve observers (age range: $18-21$ y; 3 men) participated in the experiment. Participants

99 reported normal uncorrected vision. Participants provided written informed consent prior to the 100 experiment, and received $€ 20$ for their participation. The experiment was conducted with

101 approval of the Comité d'éthique de l'Université d'Aix-Marseille (Ref.: 2014-12-03-09).

Software and apparatus

103 Eye position and pupil size were recorded monocularly with an EyeLink 1000 (SR Research,

104 Mississauga, ON, Canada), a video-based eye tracker (1000 Hz; ellipse mode). Stimuli were 105 presented on a gamma-calibrated 21" ViewSonic p227f CRT monitor (1024x768, 150 Hz).

106 Testing took take place in a dimly lit room. The experiment was implemented with OpenSesame 107 (Mathôt, Schreij, \& Theeuwes, 2012) using the PsychoPy back-end (Peirce, 2007) for display 108 control, and PyGaze (Dalmaijer, Mathôt, \& Van der Stigchel, 2014) for eye tracking.

\section{General stimuli and procedure}

110 At the beginning of each session, a nine-point eye-tracker calibration was performed. Before

111 each trial, a small dot was presented $8.5^{\circ}$ left of, right of, above, or below the display center.

112 When a stable fixation was detected, a one-point recalibration (drift correction) was performed.

113 Next, the trial started with the presentation of a green fixation dot $\left(36.8 \mathrm{~cd} / \mathrm{m}^{2}\right)$ at the same

114 location as the drift-correction stimulus. At the same time, another green dot (the saccade target)

115 was presented at the location opposite from the fixation dot (Figure 1$)$. After $1 \mathrm{~s}$, an auditory cue 116 (a $100 \mathrm{~ms}, 440 \mathrm{~Hz}$ sine wave) instructed the participant to make a saccade from the initial

117 fixation dot to the saccade target. The trial ended $3 \mathrm{~s}$ after the onset of the auditory cue.

118 Participants were instructed not to move their eyes before the cue, and to fixate on the saccade 119 target until the end of the trial. In total, the experiment lasted about 2.5 hours. For some 
120 participants, data collection was spread across two days, in which case peak saccade velocities

121 (as described below) were determined again on the second day.

[FIGURE 1]

123

124

125

126

127

128

129

130

131

132

133

134

135

136

137

138

139

140

141 142 maximum brightness $\left(95.1 \mathrm{~cd} / \mathrm{m}^{2}\right)$ had the same mean (fused) luminance as a pixel that was

\section{Part 1: Peak saccade velocity}

In the first part of the experiment, we determined the median peak velocity of saccades for each direction (left, right, down, up) and participant. The background was static and uniformly gray $\left(51.1 \mathrm{~cd} / \mathrm{m}^{2}\right)$. Gaze position was sampled on every frame (i.e. every $\left.6.67 \mathrm{~ms}\right)$. Blinks were treated as missing data. On every trial, the maximum of the peaks of the horizontal or vertical velocity profiles for the entire trial was taken as the peak saccade velocity; that is, peak velocity was the highest speed of the eyes at any moment and in any direction. When peak saccade velocity was unrealistic (above $664 \%$ s or below $220 \%$ s, based on a peak velocity of around $400 \%$ s for $17^{\circ}$ saccades, see Baloh, Sills, Kumley, \& Honrubia, 1975), the trial was discarded and repeated at a random moment during the remainder of the session. For every saccade direction, peak saccade velocity was based on the median of 40 trials.

\section{Part 2: Intrasaccadic perception}

[FIGURE 2]

In the second part of the experiment, we investigated the effect of intrasaccadic perception on pupil size. The background was a $22.6^{\circ} \times 22.6^{\circ}$ full-contrast sinusoid $\left(5.2 \mathrm{~cd} / \mathrm{m}^{2}\right.$ to $\left.95.1 \mathrm{~cd} / \mathrm{m}^{2}\right)$ grating that reversed in polarity on every frame (Figure 2). During fixation, the background appeared a homogeneous gray surface, because the frame rate $(150 \mathrm{~Hz})$ exceeded the flicker fusion threshold (see Figure 3a). Furthermore, the display appeared uniformly gray, because the monitor was gamma calibrated: A pixel that alternated between minimum $\left(5.2 \mathrm{~cd} / \mathrm{m}^{2}\right)$ and 
143 constantly at $50 \%$ brightness $\left(50.2 \mathrm{~cd} / \mathrm{m}^{2}\right)$. The spatial frequencies of the gratings were set for

144 each participant and saccade direction separately, based on the peak saccade velocities estimated

145 during the first part of the experiment. (Due to a technical issue, for three participants the gratings

146 were set to a default spatial frequency of 0.17 cycles $/{ }^{\circ}$. However, this default value was based on

147 pilot testing, and was close to the ideal spatial frequency.) The spatial frequency was such that the

148 maximum distance that the eyes traveled between two frames was equal to half a cycle. The logic

149 behind this is that the flickering background contains ambiguous motion: It can be perceived as

150 moving leftward or rightward with a speed of half a cycle per frame (Figure 2). Therefore, the

151 peak velocity of the eyes matched the velocity of the motion. As a result, the background

152 approximated a retinotopically stabilized grating when saccade velocity was maximal (igure $153 \underline{3 b})$.

[FIGURE 3]

155 In the Intrasaccadic-Percept condition, the orientation of the grating was perpendicular to the

156 direction of the saccade. Therefore, in this condition, participants should briefly perceive a static

157 grating during the saccade, as described above (Figure $3 \mathrm{~b}$ ). In the No-Percept condition, the

158 orientation of the grating was parallel with the direction of the saccade. Therefore, in this

159 condition, no static grating should be perceived, because the saccade did not stabilize the grating 160 on the retina (Figure $3 \mathrm{c})$.

\section{Part 3: Subjective report}

162 At the end of the experiment, participants provided a subjective report of how strongly they

163 had perceived 'something odd' while making saccades. They provided separate ratings for each

164 saccade direction on a 1 - 5 scale. Participants did not report the nature of the percept, 165 i.e. whether they had perceived static or moving gratings. 
168 All signals were locked to the moment that the eyes crossed the vertical (for horizontal 169 saccades) or horizontal (for vertical saccades) meridian (from now on: mid-saccade point). Mean

170 pupil size from 105 to $95 \mathrm{~ms}$ before the mid-saccade point was takes as a baseline, and all pupil 171 size measures are reported in area (i.e. not diameter) relative to this baseline (cf. Mathôt, van der

172 Linden, Grainger, \& Vitu, 2013). We analyzed pupil size from $300 \mathrm{~ms}$ before until $1200 \mathrm{~ms}$ after 173 the mid-saccade point. Pupil size during blinks was reconstructed using cubic-spline interpolation 174 (Mathôt, 2013). Pupil size was not smoothed. To obtain an acceptable noise level in eye-velocity 175 profiles, eye position was smoothed using an $11 \mathrm{~ms}$ Hanning window.

176 Trial-exclusion criteria

177 Trials were discarded when any of the following criteria was met: Saccade latency was 178 below $0 \mathrm{~ms}$ (i.e. anticipation) or above $2000 \mathrm{~ms}$; Peak saccade velocity could not be determined 179 or was unrealistically high ( $>1000 \%$ s; usually due to data loss); The eyes deviated more than $1803.3^{\circ}$ from the fixation dot before the saccade (excluding a $200 \mathrm{~ms}$ around the mid-saccade point).

181 The eyes deviated more than $3.3^{\circ}$ from the saccade target after the saccade (again excluding a $182200 \mathrm{~ms}$ around the mid-saccade point). No participants were excluded. In total, 4001 trials $183(83.3 \%)$ remained for further analysis.

\section{Position artifacts in pupil size}

185 In video-based eye trackers, changes in eye position cause artifactual changes in measured 186 pupil size. That is, when the eyes move, the angle from which the camera records the eye 187 changes, and this may lead the eye tracker to register changes in pupil size, even when pupil size 


\section{PeerJ Reviewing Manuscript}

188

189

190

191

192

193

194

remains constant. A common way to correct for position artifacts is to determine a linear regression that predicts (baseline) pupil size from horizontal $(X)$ and vertical $(Y)$ eye position. The $X$ and $Y$ slopes can then be used to 'regress out' eye position from pupil size (e.g. Brisson et al., 2013).

In our data, position artifacts are visible as rapid changes in pupil size during the saccade (Figure 5a,d,j,g); these changes are too rapid to be real. But when we tried to eliminate position artifacts through linear regression, we noticed the following: When fixating the top of the screen, the pupil was larger than when fixating the bottom of the screen (data not shown), which suggests that pupil size is overestimated when fixating the top of the screen. But when participants made a top-to-bottom saccade, pupil size increased even further (Figure 5j), which suggests that pupil size is underestimated when fixating the top of the screen. In other words, the direction of the position artifact seems to depend on whether you look at baseline pupil size, or at the rapid change that occurs during the saccade. This implies that there are two distinct effects of eye position on pupil size as measured by the eye tracker: The first is a real effect, so that (in our case) the pupil slightly dilates when fixating the top of the screen, perhaps due to effort or the eye's musculature; the second is an artifact, so that (in our case) the size of the pupil is underestimated when fixating the top of the screen. We are currently investigating this issue further, but for now we note that: not all effects of eye position on pupil size are artifactual (as generally assumed); and any corrective technique that assumes this is problematic.

Therefore, we decided not to correct our pupil-size measurements for position artifacts, and absolute changes in pupil size that occur while the eyes are moving (as shown in Figure 5a,d,j,g) should be interpreted with caution.

\section{Statistical analyses}

Unless otherwise specified, we used linear mixed-effects models (LME) with by-participant 
212 random intercept and by-participant random slopes for all predictors. Identical analyses were

213 conducted separately for each $1 \mathrm{~ms}$ sample. We did not estimate $p$-values, but considered effects

214 reliable if they correspond to $t>2$ for at least 200 consecutive $1 \mathrm{~ms}$ samples (cf. Mathôt,

215 Dalmaijer, Grainger, \& Van der Stigchel, 2014). However, we emphasize effect sizes and overall 216 patterns.

Results

\section{Differences between saccade directions}

\section{Intrasaccadic perception induced pupillary constriction}

The main result is shown in Figure 4a, in which pupil size is plotted over time as a function of condition (Intrasaccadic Percept vs No Percept). The pupil initially dilated in both conditions, until about $200 \mathrm{~ms}$ after the mid-saccade point, reflecting motor preparation (Jainta, Vernet, Yang, \& Kapoula, 2011; Wang, Brien, \& Munoz, 2015). Next, the pupil constricted in both conditions from about $220 \mathrm{~ms}$ after the mid-saccade point. Such a constriction is typically observed after saccades (Mathôt et al., 2015; Zuber et al., 1966). Crucially, as predicted, this constriction was more pronounced in the Intrasaccadic-Percept Condition than in the No-Percept Condition. Based on an LME with pupil size as dependent measure, and Condition (reference: No Percept), Saccade Direction (reference: Horizontal), and their interaction as fixed effects, the effect of condition was reliable from $306 \mathrm{~ms}$ after the mid-saccade point until the end of the analysis period. As can be seen in the indivual-participant data (Figure 4b), this effect was highly consistent across participants.

\section{[FIGURE 4]}

Based on the LME described above, there was a reliable interaction between Condition and Saccade Direction from 426 ms until the end of the analysis period. This interaction indicated 
235 that the Condition effect was driven mainly by horizontal saccades. We confirmed this by 236 analyzing each saccade direction separately, based on four separate LMEs with Condition as

237 fixed effect and pupil size as dependent measure (Figure 5). There was a strong and reliable

238 Condition effect for leftward and rightward saccades (Figure 5a,d). There was also a reliable

239 Condition effect for downward saccades (Figure 5g), but it was much weaker. For upward 240 saccades, there was no reliable Condition effect (see Figure 5j). In addition, there was

241 considerable sample-to-sample variability in the model estimates for upwards saccades (as shown

242 by the jittery error bands in Figure $5 \mathrm{j}$ ), suggesting that this subset of data was especially noisy.

[FIGURE 5]

244 Although there are known differences between saccades in different directions (Collewijn,

245 Erkelens, \& Steinman, 1988; Van der Stigchel \& Theeuwes, 2008), we had not expected saccade

246 direction to interact with the effect of intrasaccadic perception on pupil size. Therefore, we

247 conducted several post-hoc analyses to better understand these differences.

248 First, peak velocities are more variable for vertical than horizontal saccades (compare Figure

$249 \underline{5} \mathrm{c}, \mathrm{f}, \mathrm{i}, \mathrm{l})$. This was the case for all participants (two-sided paired-samples t-test using per-

250 participant standard deviation of peak velocity as dependent measure: $t(9)=6.42, p=.0001$ ).

251 This variability is important, because the intrasaccadic percept was optimized for a peak velocity

252 of around $400 \%$ s (depending slightly on the participant and saccade direction). Therefore, the

253 intrasaccadic percept may have been less salient for vertical than horizontal saccades. To control

254 for this, we determined the difference between the actual and the optimal peak velocity (from

255 now on: peak-velocity error) for each trial. Next, we selected trials on which peak-velocity error

256 was less than the median peak-velocity error (separately for each participant and saccade

257 direction).

258 Second, vertical saccades are more curved than horizontal saccades (Van der Stigchel \& 259 Theeuwes, 2008). Therefore, the velocity component that is perpendicular to the saccade 
260 direction (i.e. horizontal velocity during vertical saccades, and vertical velocity during horizontal

261 saccades; from now on: orthogonal velocity) was higher for vertical than horizontal saccades

262 (Figure 6). This was the case for all participants (two-sided paired-samples t-test using per-

263 participant mean peak orthogonal velocity as dependent measure: $t(9)=10.25, p<.0001)$. Peak

264 orthogonal velocity during vertical saccades often approached $150^{\circ} / \mathrm{s}$. In the No Percept

265 condition, this would have led to partial retinal stabilization of the grating, which is sufficient to

266 trigger an intrasaccadic percept (García-Pérez \& Peli, 2001). In other words, intrasaccadic

267 perception may have triggered a pupillary constriction in both conditions, thus reducing the

268 Condition effect. To control for this, we selected trials on which peak orthogonal velocity was

269 less than the median peak orthogonal velocity (seperately for each participant and saccade

270 direction).

[FIGURE 6]

272 Next, using vertical saccades from this subset of data, selected as described above, we

273 performed the same analysis as before. Based on an LME with Condition as fixed effect and

274 pupil size as dependent measure, we now also observed a reliable Condition effect for vertical

275 saccades (Figure 7). Therefore, it is likely that the Condition effect for vertical saccades was

276 reduced by variability in peak velocity, and pronounced curvature.

277

[FIGURE 7]

278

279

280

281

282

283

\section{Subjective ratings}

Figure 8 shows the participants' subjective ratings of how strongly they had perceived 'something odd' during saccades, separately for each saccade direction. These ratings suggest that all participants experienced an intrasaccadic percept, despite the fact that all participants were naïve and intrasaccadic perception was not mentioned until the end of the experiment. There is a tendency for the intrasaccadic percept to be rated more salient for horizontal than vertical 
284 saccades, but this tendency is weak and unreliable $(F(3,27)=0.918, p=.446$; based on a

285 Repeated Measures Analysis of Variance with saccade direction as independent variable and 286 rating as dependent measure).

Here we report that intrasaccadic perception (seeing while the eyes move) triggers a constriction of the eye's pupil. We presented participants with sinusoid gratings that changed in

291 polarity 150 times per second, or every $6.67 \mathrm{~ms}$. At this speed, successive frames cannot be

292 distinguished, and the flickering gratings were therefore perceived as homogeneous gray surfaces

293 (Figure 3a). However, the flickering gratings contained two opposite motion signals (Figure 2). In

294 one condition, there was a brief moment during each saccade at which the eye's velocity matched

295 the direction and speed of one of the grating's motion signals (Figure $3 b$ ). This resulted in a 296 stable retinal image, in turn resulting in a brief-but-clear intrasaccadic percept. Relative to a 297 control condition in which the saccade direction did not match the motion signal, this 298 intrasaccadic percept triggered a slight pupillary constriction (Figure 4).

299 To our knowledge, our study provides the first direct evidence for intrasaccadic perception 300 that does not rely on subjective report. We did collect subjective ratings, which confirmed that 301 participants saw an intrasaccadic percept (Figure 8), but these ratings were collected after the

302 experiment. Furthermore, our results show that intrasaccadic perception affects all stages of 303 visual processing: from the reflexive pupillary response, as we report here, to conscious 304 perception, as reported previously (Campbell \& Wurtz, 1978; Castet \& Masson, 2000; Castet, 305 Jeanjean, \& Masson, 2002; Deubel \& Elsner, 1986; García-Pérez \& Peli, 2001). The picture that 306 emerges is that there is nothing special about intrasaccadic perception. Visual perception may not

307 be fundamentally different when the eyes move, compared to when the eyes do not move. 
308 So far, we have focused on the pupillary constriction that is triggered by intrasaccadic

309 perception (i.e. the difference between lines in Figure 4). But saccades are usually followed by a

310 pronounced constriction, also without intrasaccadic perception (i.e. the overall negative

311 deflection in Figure 4). Zuber and colleagues $(\underline{1966})+($; see also Mathôt et al., 2015) already

312 noticed this, but attributed it to changes in accommodation that might accompany gaze shifts

313 (i.e. the pupil near reflex). However, based on recent(ish) advances in pupillometry, we suggest

314 that post-saccadic pupillary constriction may directly reflect intrasaccadic perception-both when

315 consciously perceived, and when not consciously perceived.

316 As first documented by Van de Kraats, Smit, and Slooter (1977), any kind of change in

317 visual input triggers a pupillary constriction, even when overall brightness does not change. For

318 example, when you look at a checkerboard that changes polarity (the dark squares become bright,

319 and vice versa), your pupil briefly constricts, before returning to its normal size (Slooter \& van

320 Norren, 1980; Ukai, 1985). The origin of this phenomenon is unclear. According to Barbur,

321 Keenleyside, and Thompson (1987) $+($; cited in Sahraie \& Barbur, 1997), it may result from

322 changes in neural activity that occur when visual input changes. This may cause a temporary

323 reduction of the inhibition of the pathway that drives the pupillary sphincter muscle, thus causing

324 a brief constriction. Alternatively, as we have recently suggested, pupillary constriction to visual

325 change may simply reflect the asymmetry of the pupillary light response (Mathôt et al., 2013):

326 Dilation in response to darkness is slower than constriction in response to brightness. Because

327 visual changes are generally a mix of local increases and decreases in brightness, the combined

328 pupillary response may be an initial constriction (to local brightness increases) that disappears as

329 dilation (to local brightness decreases) catches up. But, whatever the cause, it is clear that the

330 pupil constricts in response to changes in visual input.

331 Our interpretation of the postsaccadic pupillary response (Figure 4) is therefore as follows.

332 We assume that pupillary constriction to visual change is largely reflexive, but can be modulated 
333 by top-down factors, such as visual awareness. When the eyes move, visual input changes, and

334 this triggers a pupillary constriction, just like a checkerboard inversion does. Usually, these

335 saccade-related visual changes are not consciously perceived; but when they are, visual

336 awareness increases the pupillary constriction. In other words, the slight additional constriction

337 that is triggered by intrasaccadic perception may reflect top-down enhancement of the pupillary

338 constriction to visual change. Although this interpretation is speculative, it fits with recent

339 findings that pupillary responses, even those that were traditionally seen as pure reflexes, are

340 susceptible to top-down modulation (reviewed in Mathôt \& Van der Stigchel, 2015).

341 In summary, we have shown that intrasaccadic perception triggers a pupillary constriction, or

342 rather strengthens the pupillary constriction that is generally observed after saccadic eye

343 movements. To our knowledge, this is the first direct evidence for intrasaccadic perception that

344 does not rely on subjective report: Regardless of whether you expect it or not, intrasaccadic

345 perception is a salient event that affects all stages of visual processing, from the pupillary

346 response to visual awareness. 
348 Baloh, R. W., Sills, A. W., Kumley, W. E., \& Honrubia, V. (1975). Quantitative measurement of

349 saccade amplitude, duration, and velocity. Neurology, 25(11), 1065-1065.

$350 \quad$ http://doi.org/10.1212/wnl.25.11.1065

351 Barbur, J. L., Keenleyside, M. S., \& Thomson, W. D. (1987). Investigation of central visual 352 processing by means of pupillometry. In Seeing Contour and Colour: Proceedings of the 3rd 353 International Symposium of the Northern Eye Institute (pp. 431-451). Oxford, UK: $354 \quad$ Pergamon Press.

355 Binda, P., \& Murray, S. O. (2014). Keeping a large-pupilled eye on high-level visual processing. 356 Trends in Cognitive Sciences, 19(1), 1-3. http://doi.org/10.1016/j.tics.2014.11.002

357 Braun, D., Schuetz, C. S., \& Gegenfurtner, K. R. (2015). Changes in visual sensitivity during 358 smooth pursuit and saccadic eye movements. In Journal of Vision. St. Pete Beach, FL, USA.

359 Bremmer, F., Kubischik, M., Hoffmann, K., \& Krekelberg, B. (2009). Neural Dynamics of 360 Saccadic Suppression. The Journal of Neuroscience, 29(40), 12374-12383. $361 \quad$ http://doi.org/10.1523/jneurosci.2908-09.2009

362 Brisson, J., Mainville, M., Mailloux, D., Beaulieu, C., Serres, J., \& Sirois, S. (2013). Pupil 363 diameter measurement errors as a function of gaze direction in corneal reflection eyetrackers. 364 Behavior Research Methods, 45(4), 1322-1331. http://doi.org/10.3758/s13428-013-0327-0

365 Burr, D. C., Morrone, M. C., \& Ross, J. (1994). Selective suppression of the magnocellular visual 366 pathway during saccadic eye movements. Nature, 371(6497), 511-513. $367 \quad$ http://doi.org/10.1038/371511a0

368 Campbell, F. W., \& Wurtz, R. H. (1978). Saccadic omission: why we do not see a grey-out during 369 a saccadic eye movement. Vision Research, 18(10), 1297-1303. http://doi.org/10.1016/0042$370 \quad \underline{6989(78) 90219-5}$ 
371 Castet, E. (2010). Perception of intra-saccadic motion. In U. J. Ilg \& G. S. Masson (Eds.), 372 Dynamics of Visual Motion Processing (pp. 213-238). Springer Science.

373 Castet, E., \& Masson, G. S. (2000). Motion perception during saccadic eye movements. Nature

374 Neuroscience, 3(2), 177-183. http://doi.org/10.1038/72124

375 Castet, E., Jeanjean, S., \& Masson, G. S. (2002). Motionperception of saccade-induced retinal

376 translation. Proceedings of the National Academy of Sciences, 99(23), 15159-15163.

377 http://doi.org/10.1073/pnas.232377199

378 Collewijn, H., Erkelens, C. J., \& Steinman, R. M. (1988). Binocular co-ordination of human

379 horizontal saccadic eye movements. The Journal of Physiology, 404(1), 157-182.

$380 \quad$ http://doi.org/10.1113/jphysiol.1988.sp017284

381 Cousineau, D. (2005). Confidence intervals in within-subject designs: A simpler solution to 382 Loftus and Masson's method. Tutorial in Quantitative Methods for Psychology, 1(1), 4-45.

383 Dalmaijer, E., Mathôt, S., \& Van der Stigchel, S. (2014). PyGaze: An open-source, cross-platform

384 toolbox for minimal-effort programming of eyetracking experiments. Behavior Research 385 Methods, 46(4), 913-921. http://doi.org/10.3758/s13428-013-0422-2

386 Deubel, H., \& Elsner, T. (1986). Threshold perception and saccadic eye movements. Biological 387 Cybernetics, 54(6), 351-358. http://doi.org/10.1007/bf00355540

388 Diamond, M. R., Ross, J., \& Morrone, M. C. (2000). Extraretinal control of saccadic suppression. 389 The Journal of Neuroscience, 20(9), 3449-3455.

390 Gamlin, P. D. R., Zhang, H., Harlow, A., \& Barbur, J. L. (1998). Pupil responses to stimulus

391 color, structure and light flux increments in the rhesus monkey. Vision Research, 38(21), 392 3353-3358. http://doi.org/10.1016/s0042-6989(98)00096-0

393 García-Pérez, M. A., \& Peli, E. (2001). Intrasaccadic perception. The Journal of Neuroscience, $39421(18), 7313-7322$.

395 Jainta, S., Vernet, M., Yang, Q., \& Kapoula, Z. (2011). The pupil reflects motor preparation for 
saccades - even before the eye starts to move. Frontiers in Human Neuroscience, 5. http://doi.org/10.3389/fnhum.2011.00097

398 Knöll, J., Binda, P., Morrone, M. C., \& Bremmer, F. (2011). Spatiotemporal profile of peri-

399 saccadic contrast sensitivity. Journal of Vision, 11(14), 15. http://doi.org/10.1167/11.14.15

400 Lorber, M., Zuber, B. L., \& Stark, L. (1965). Suppression of the pupillary light reflex in binocular 401 rivalry and saccadic suppression. Nature, 208, 558-560. http://doi.org/10.1038/208558a0

402 Mathôt, S. (2013). A Simple Way to Reconstruct Pupil Size During Eye Blinks. Retrieved from $403 \quad$ http://dx.doi.org/10.6084/m9.figshare.688001

404 Mathôt, S., \& Van der Stigchel, S. (2015). New light on the mind's eye: The pupillary light 405 response as active vision. Current Directions in Psychological Science.

406 Mathôt, S., Dalmaijer, E., Grainger, J., \& Van der Stigchel, S. (2014). The pupillary light 407 response reflects exogenous attention and inhibition of return. Journal of Vision, 14(14), 7. $408 \quad$ http://doi.org/10.1167/14.14.7

409 Mathôt, S., Schreij, D., \& Theeuwes, J. (2012). OpenSesame: An open-source, graphical 410 experiment builder for the social sciences. Behavior Research Methods, 44(2), 314-324. $411 \quad$ http://doi.org/10.3758/s13428-011-0168-7

412 Mathôt, S., van der Linden, L., Grainger, J., \& Vitu, F. (2013). The pupillary response to light 413 reflects the focus of covert visual attention. PLoS ONE, 8(10), e78168. $414 \quad$ http://doi.org/10.1371/journal.pone.0078168

415 Mathôt, S., van der Linden, L., Grainger, J., \& Vitu, F. (2015). The pupillary light response 416 reflects eye-movement preparation. Journal of Experimental Psychology: Human Perception 417 and Performance, 41(1), 28-35. http://doi.org/10.1037/a0038653

418 O'Regan, J. K., \& Noë, A. (2001). A sensorimotor account of vision and visual consciousness. 419 Behavioral and Brain Sciences, 24(5), 939-1031. $420 \quad$ http://doi.org/10.1017/s0140525x01000115 
421 Peirce, J. W. (2007). PsychoPy: Psychophysics software in Python. Journal of Neuroscience

422 Methods, 162(1-2), 8-13. http://doi.org/10.1016/j.jneumeth.2006.11.017

423 Ross, J., Burr, D. C., \& Morrone, C. (1996). Suppression of the magnocellular pathway during

424 saccades. Behavioural Brain Research, 80(1-2), 1-8. http://doi.org/10.1016/0166-

$425 \quad \underline{4328(96) 00012-5}$

426 Sahraie, A., \& Barbur, J. L. (1997). Pupil response triggered by the onset of coherent motion.

427 Graefe's Archive for Clinical and Experimental Ophthalmology, 235(8), 494-500. 428 http://doi.org/10.1007/bf00947006

429 Slooter, J. H., \& van Norren, D. (1980). Visual acuity measured with pupil responses to $430 \quad$ checkerboard stimuli. Investigative Ophthalmology \& Visual Science, 19(1), 105-108.

431 Thiele, A., Henning, P., Kubischik, M., \& Hoffmann, K. P. (2002). Neural mechanisms of 432 saccadic suppression. $\quad$ Science, 2460-2462. $433 \quad$ http://doi.org/10.1126/science. 1068788

434 Ukai, K. (1985). Spatial pattern as a stimulus to the pupillary system. Journal of the Optical 435 Society of America A, 2(7), 1094-1100. http://doi.org/10.1364/josaa.2.001094

436 Van de Kraats, J., Smit, E. P., \& Slooter, J. H. (1977). Objective perimetric measurements by the 437 pupil balance method. In Second International Visual Field Symposium, Tubingen, 19-22 438 September, 1976 (pp. 213-220). Springer Science \& Business Media.

439 Van der Stigchel, S., \& Theeuwes, J. (2008). Differences in distractor-induced deviation between 440 horizontal and vertical saccade trajectories: NeuroReport, 19(2), 251-254. $441 \quad$ http://doi.org/10.1097/wnr.0b013e3282f49b3f

442 Volkmann, F. C., Riggs, L. A., White, K. D., \& Moore, R. K. (1978). Contrast sensitivity during 443 saccadic eye movements. Vision Research, 18(9), 1193-1199. http://doi.org/10.1016/0042$444 \quad \underline{6989(78) 90104-9}$

445 Wang, C., Brien, D. C., \& Munoz, D. P. (2015). Pupil size reveals preparatory processes in the 
446 generation of pro-saccades and anti-saccades. European Journal of Neuroscience. $447 \quad$ http://doi.org/10.1111/ejn.12883

448 Zuber, B. L., \& Stark, L. (1966). Saccadic suppression: Elevation of visual threshold associated 449 with saccadic eye movements. Experimental Neurology, 16(1), 65-79. $450 \quad$ http://doi.org/10.1016/0014-4886(66)90087-2

451 Zuber, B. L., Stark, L., \& Lorber, M. (1966). Saccadic suppression of the pupillary light reflex. 452 Experimental Neurology, 14(3), 351-370. http://doi.org/10.1016/0014-4886(66)90087-2 
Figure 1 (on next page)

Schematic experimental paradigm

Participants initially fixated on a dot presented at the left, right, top, or bottom of the screen. A saccade target was presented at the location opposite from the fixation dot. An auditory cue instructed participants to make a saccadic eye movement to the saccade target. 


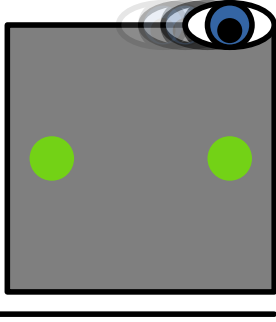

$1 \mathrm{~s}$

$3 s$ 
Figure 2 (on next page)

Illustration of ambiguous motion

During Part 2 of the experiment (see main text), the background consisted of a sinusoid grating that reversed polarity on every frame. The motion direction in this flickering display is ambiguous. The two opposite motion signals (leftward: red arrow; rightward: green arrow) result from matching the white and black bars across time. When viewed with static eyes, the flickering display appears as a homogeneous gray surface. 


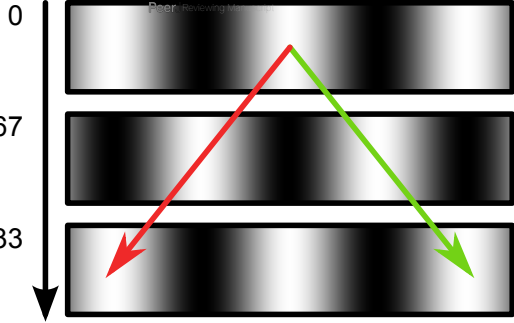

13.33

Perceived leftward motion Perceived rightward motion 
Figure $\mathbf{3}$ (on next page)

Illustration of intrasaccadic perception

a) The background is a rapidly flickering grating that is perceived as uniformly gray during fixation. b) When a saccade is made perpendicular to the grating (Intrasaccadic-Percept condition), the grating is briefly stabilized on the retina, which results in a flash-like intrasaccadic percept. c) When a saccade is made parallel to the grating (No-Percept condition), the grating is not stabilized on the retina, and no intrasaccadic percept arises. 
Figure 4 (on next page)

Main results

a) Mean normalized pupil size over time, locked to the mid-saccade point. There was a more pronounced constriction in the Intrasaccadic Percept Condition than in the No-Percept Condition. Error bands indicate the standard error. Gray shading indicates a reliable effect of Condition. b) The pupil-size difference between the Intrasaccadic Percept and No-Percept conditions for each individual participant. 


\section{Figure 5 (on next page)}

Results split by saccade direction

Pupil-size, eye-position, and eye-velocity traces for each of the four saccade directions. The large differences in pupil size just before and after saccades between the four saccade directions are due to position artifacts in pupil-size measurements (see main text). a,d,g,j) Error bands indicate the standard error. Gray shading indicates a reliable effect of condition. See main text for a description of the statistical models. b,e) Horizontal eye position over time. $c, f)$ Horizontal eye velocity over time. $h, k$ ) Vertical eye position over time. i,l) Vertical eye velocity over time. $b, c, e, f, h, i, k, l)$ Individual trials are color coded to indicate peak saccade velocity (red: high velocity; blue: low velocity). 
Figure $\mathbf{6}$ (on next page)

Orthogonal eye velocity

Eye velocity perpendicular to the saccade direction (orthogonal velocity). a, b) Vertical eye velocity during horizontal saccades. c,d) Horizontal velocity during vertical saccades. Individual trials are color coded to indicate peak orthogonal velocity (red: high velocity; blue: low velocity). 


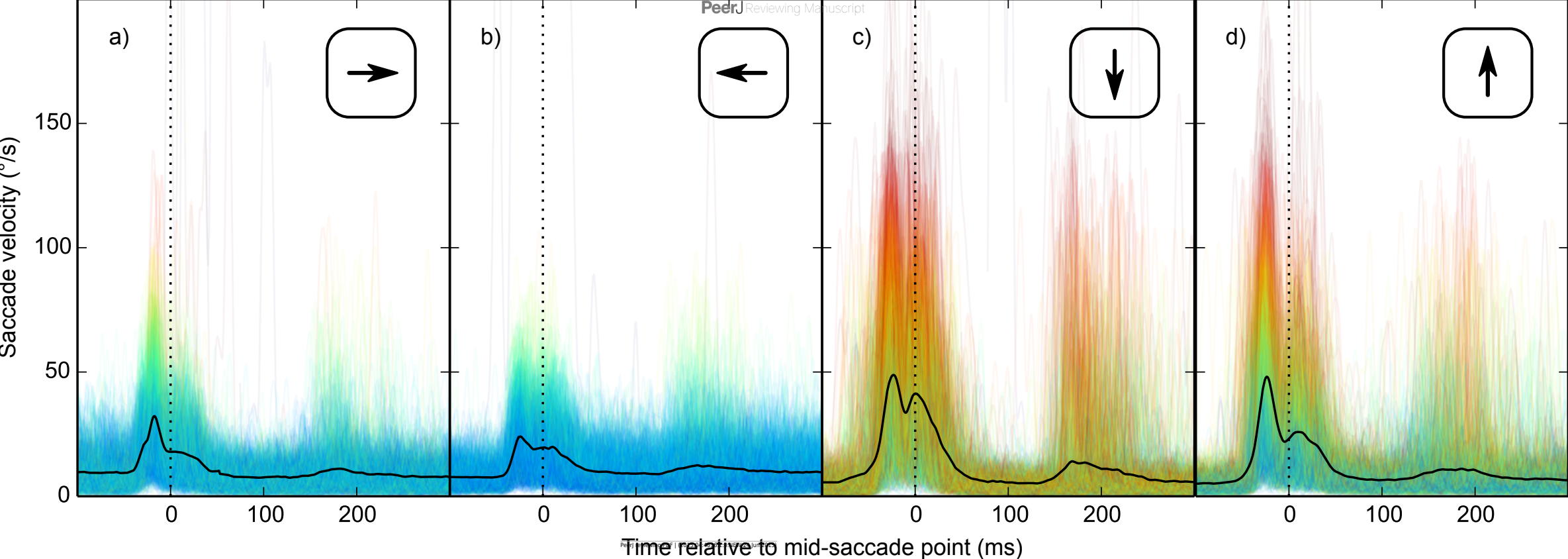


Figure 7 (on next page)

Results for an optimal subset of vertical saccades

Analysis of a subset of vertical-saccade trials on which orthogonal velocity was low and peak saccade velocity was close to optimal. Pupil size is plotted over time, locked to the midsaccade point. There was a more pronounced constriction in the Intrasaccadic Percept Condition than in the No-Percept Condition. Error bands indicate the standard error. Gray shading indicates a reliable effect of Condition. 


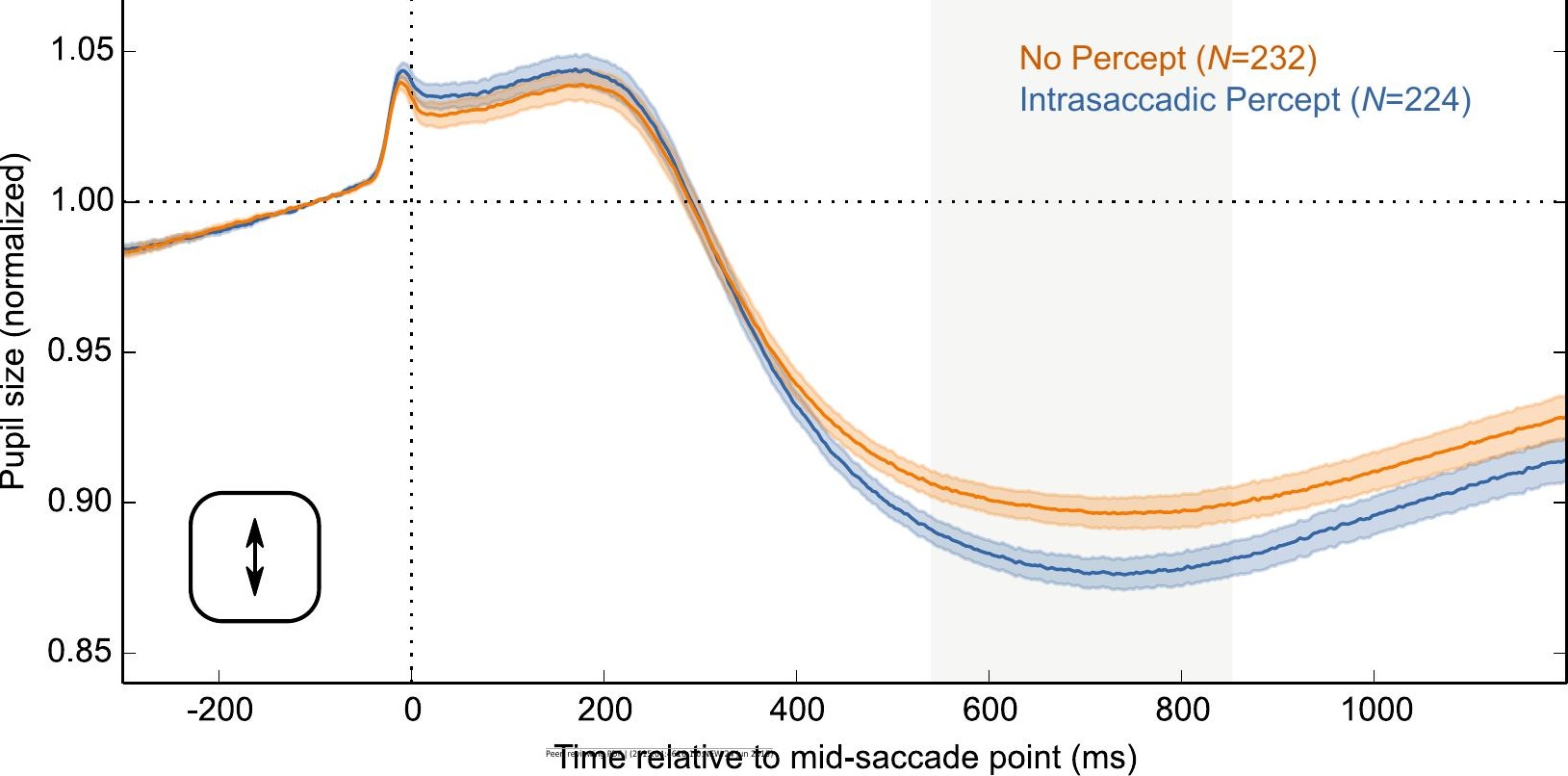


Figure 8 (on next page)

Subjective ratings of intrasaccadic perception

Participants' ratings of how strongly they had perceived 'something odd' during saccades, separately for each saccade direction. Error bars indicate 95\% within-subject confidence intervals (Cousineau, 2005). Dots correspond to single ratings, color coded by participant. 
PeerJReviewing Manuscript

\section{Very}

clearly 5 